\title{
LA IMPORTANCIA DE LA GESTIÓN AMBIENTAL MUNICIPAL. ESTUDIO DE CASO: MUNICIPIOS DEL DEPARTAMENTO DE SANTA ANA, EL SALVADOR
}

\author{
THE IMPORTANCE OF MUNICIPAL ENVIRONMENTAL MANAGEMENT. A CASE STUDY OF \\ MUNICIPALITIES IN THE DEPARTMENT OF SANTA ANA, EL SALVADOR \\ A IMPORTÂNCIA DO GERENCIAMENTO AMBIENTAL MUNICIPAL. ESTUDO DE CASO: \\ MUNICÍPIOS DO DEPARTAMENTO DE SANTA ANA, EL SALVADOR \\ Bismarck Toledo ${ }^{1}$
}

Fecha de recibido: Agosto 06 de 2017 | Fecha de aprobado: Octubre 03 de 2017

\section{Resumen}

La investigación titulada "La importancia de la gestión ambiental municipal. Caso de estudio municipios en el departamento de Santa Ana, El Salvador" tuvo por objetivo general realizar una evaluación crítica y constructiva del tema de la administración local en materia ambiental específicamente en las municipalidades de los distritos del departamento de Santa Ana, en las cuales se estudiaron los siguientes aspectos relevantes: análisis de los conceptos básicos e historia de la gestión ambiental en El Salvador; análisis de la legislación y marco regulatorio existente en materia ambiental, normativas nacionales de obligatorio cumplimiento así como de las normativas y estándares internacionales de cumplimento voluntario sobre la gestión ambiental municipal. Se estableció si la infraestructura, equipo y el personal es el adecuado para el desarrollo óptimo de la unidad ambiental; posteriormente, se analizó si las unidades ambientales cuentan con los instrumentos administrativos básicos necesarios para cumplir con la misión que la Ley de Medio Ambiente le ha delegado.

Luego de analizar cada uno de estos factores, se llega a la conclusión de que en general las municipalidades han realizado esfuerzos para afrontar la problemática ambiental pero la falta de gestión ambiental local eficiente hacen que estos esfuerzos no sean suficientes, no es extraño que los gobiernos municipales no dispongan de los instrumentos conceptuales, administrativos y operativos necesarios para la incorporación de la perspectiva ambiental en las actividades que se realizan dentro del municipio, lo que conlleva un peligroso y acelerado detrimento del medio ambiente.

Palabras claves: Gestión ambiental, gestión ambiental local, unidad ambiental municipal, sistema de gestión ambiental, desarrollo sostenible.

1 Docente investigador de la Universidad Católica de El Salvador, Unicaes.

Correo electrónico: bismarcktoledo@hotmail.com 


\section{Abstract}

The purporse of the research "The importance of municipal environmental management. A case study of municipalities in the department of Santa Ana, El Salvador" was to conduct a critical and constructive assessment of local environmental management, particularly in the municipalities of the districts of the department of Santa Ana. Some of the issues addressed were: analysis of basic concepts and a history of environmental management in El Salvador; analysis of the legislation and the normative framework in environmental issues, mandatory national norms, as well as non-mandatory international norms and standards on municipal environmental management. The authors established whether the current infrastructure, equipment and staff are appropriate to get the optimal development of the environmental units. Then, they analyzed whether those units possess the basic administrative tools necessary to comply with the Environment Law.

After studying each of these factors, it is concluded that overall the municipalities have striven to face environmental challenges. However, due to the lack of an efficient local environmental management, these efforst are not enough. It is not uncommon that municipal governments lack the conceptual, administrative and operational tools required to include the environmental approach in their activities. This entails a dangerous and hastened environmental detriment.

Keywords: Environmental management, local environmental management, municipal environmental unit, environmental management system, sustainable development.

\section{Resumo}

Na pesquisa “A importância do gerenciamento ambiental municipal. Caso de estudo municípios no departamento de Santa Ana, El Salvador" teve por objetivo geral realizar uma avaliação crítica e construtiva do tema da administração local em matéria ambiental especificamente nas municipalidades dos distritos do departamento de Santa Ana, nas quais se estudaram os seguintes aspectos relevantes: análise dos conceitos básicos e história do gerenciamento ambiental em El Salvador; análise da legislação e marco regulatório existente em matéria ambiental, regulamentos nacionais de obrigatório cumprimento bem como das normativas e padrões internacionais de cumprimento voluntário sobre o gerenciamento ambiental municipal. Estabeleceu-se se a infraestrutura, equipe e o pessoal é o adequado para o desenvolvimento ótimo da unidade ambiental; posteriormente, analisou-se se as unidades ambientais contam com os instrumentos administrativos básicos necessários para cumprir com a missão que a Lei de Meio ambiente the tem delegado.

Após de analisar a cada um destes fatores, se chega à conclusão de que em geral as municipalidades têm realizado esforços para enfrentar a problemática ambiental mas a falta de gerenciamento ambiental local eficiente fazem que estes esforços não sejam suficientes, não é estranho que os governos municipais não disponham dos instrumento conceituais, administrativos e operativos necessários para a incorporação da perspectiva ambiental nas atividades que se realizam dentro do município, o que implica um perigoso e acelerado detrimento do meio ambiente.

Palavras-chave: Gerenciamento ambiental, gerenciamento ambiental local, unidade ambiental municipal, sistema de gerenciamento ambiental, desenvolvimento sustentável. 


\section{INTRODUCCIÓN}

Las décadas de deterioro progresivo de la calidad ambiental en El Salvador han acarreado el escalamiento de numerosos conflictos sectoriales. El crecimiento de la problemática ambiental es preocupante, se requiere de la búsqueda de alternativas de solución que conduzcan a la prevención del deterioro vertiginoso de los recursos naturales. Para proteger el medio ambiente es importante que todos los sectores del nivel nacional y local actúen sobre la problemática ambiental. Por lo tanto, los gobiernos municipales deben asumir el rol y responsabilidades correspondientes sobre la protección, conservación y manejo de los recursos naturales (Ministerio de Medio Ambiente y Recursos Naturales, MARN, 2015).

La gestión ambiental (GA) tiene la finalidad de reducir este problema, al fortalecer la presencia de la institucionalidad en materia ambiental, este es un proceso que se fundamenta en los principios de la gerencia moderna: organizar, planificar, ejecutar, dirigir y controlar. La gestión ambiental local (GAL) es un proceso descentralizador fundado en la participación ciudadana, que tiene por objeto asegurar la corresponsabilidad en la toma de decisiones ambientales. Esta gestión requiere la participación de municipios y organizaciones ciudadanas para enfrentar desde una perspectiva sistemática e integral los problemas del territorio y desarrollar soluciones válidas para cada uno de los municipios (MARN, 2015).

En El Salvador, la base legal de la gestión ambiental local se estipula en el art. 2 de la Ley de Medio Ambiente en uno de los "Principios de la Política Nacional del Medio Ambiente" en el literal h:

La gestión pública del medio ambiente debe ser global y transectorial, compartida por las distintas instituciones del Estado, incluyendo los Municipios y apoyada y complementada por la sociedad civil, de acuerdo a lo establecido por esta ley, sus reglamentos y demás leyes de la materia. (MARN, 1998).

La investigación tuvo como objetivo general analizar la situación actual de las unidades ambientales de los municipios del departamento de Santa Ana para determinar la capacidad de gestión ambiental municipal, por medio del análisis de las herramientas y procedimientos que garantizaran la sostenibilidad y participación de la comunidad. Así pues, esperamos que este trabajo contribuya tanto a crear como a fortalecer los mecanismos institucionales que hacen posible la gestión municipal en el ámbito territorial.
El rol de los gobiernos municipales en materia de gestión ambiental se encuentra sustentando en el marco legal constitucional y en la legislación secundaria de El Salvador, en el cual se establecen responsabilidades, funciones y servicios (MARN, 2015):

- Elaboración del diagnóstico, la estrategia y el plan de acción ambiental.

- Elaboración de ordenanzas ambientales y/o revisión y divulgación de las ya existentes.

- Apoyar las iniciativas locales tendientes a establecer alianzas estratégicas entre el sector municipal y empresarial, vinculadas a proteger el medio ambiente.

- Apoyar la incorporación del desarrollo económico local en la gestión pública municipal, buscando el equilibrio ambiental, social y económico.

Estas funciones y responsabilidades se ven seriamente limitadas ya que en algunos casos no existen los instrumentos administrativos de gestión o las acciones en este sentido son incipientes, como lo demuestran los resultados de la investigación.

\section{REFERENTE TEÓRICO}

A continuación, se presenta una breve exposición de los conceptos básicos para comprender la importancia de la gestión ambiental municipal.

Desarrollo sustentable: "Es aquel que puede lograr satisfacer las necesidades y las aspiraciones del presente, sin comprometer la capacidad de las generaciones futuras de satisfacer sus propias necesidades y aspiraciones" (Comisión Mundial sobre Medio Ambiente y Desarrollo, onU, 1987).

\section{Gestión ambiental:}

La gestión es un proceso continuo de carácter cíclico que comprende dos grandes etapas: la planificación y la administración. La gestión ambiental busca el desarrollo sostenible, es decir, para conseguir un equilibrio adecuado para el desarrollo económico, crecimiento de la población, uso racional de los recursos y protección y conservación del ambiente. (Ministerio de Medio Ambiente y Recursos Naturales, República Dominicana, 2012).

Gestión pública municipal: "Todas las actividades o mandatos legales querealizan o ejecutan las 
municipalidades en relación al medio ambiente con consecuencia o impacto en el mismo" (Asociación Chilena de Municipalidades, Fundación Friedrich Ebert, Santiago de Chile, 1995).

Unidad Ambiental Municipal: La presencia de la Unidad Ambiental contribuye a que los municipios se preparen para asumir nuevas atribuciones y para utilizar mejor las existentes.

Desde la perspectiva de la descentralización administrativa, la presencia del área de medio ambiente en la estructura orgánica de las municipalidades constituye un importante avance para los gobiernos locales. En este sentido, sus funciones son de: Supervisar, coordinar, dar seguimiento a las políticas, planes, programas, proyectos y acciones ambientales dentro del municipio. (MARN, 2015).

\section{Plan de Gestión Ambiental Municipal:}

Es el conjunto de propuestas organizadas de acuerdo a objetivos generales y específicos y Acciones. Estas últimas se elaboran en forma de programas y proyectos priorizados en una secuencia temporal lógica, señalando los indicadores de avance o logros, según plazos estimados. (International Organization for Standardization, Iso, 2015).

Diagnóstico ambiental del municipio: "Consiste en estudiar el conjunto de características y síntomas ambientales manifestados en una localidad. Usando esta información se genera un listado de problemas percibidos tanto en el municipio mismo como por la comunidad organizada" (Ministerio de Medio Ambiente y Recursos Naturales, República Dominicana, 2012).

Sistemas de gestión ambiental: "Destinados a permitir que una organización desarrolle e implemente una política y unos objetivos que tengan en cuenta los requisitos legales, y la información relativa a los aspectos ambientales significativos" (International Organization for Standardization, Iso, 2015).

Los países latinoamericanos en general han realizado esfuerzos importantes por establecer una gestión ambiental local más fuerte entre algunas estas experiencias podemos mencionar la siguiente:

- El caso República Dominicana, “El programa de Fortalecimiento de las Unidades de Gestión Ambiental Municipal". Bajo los auspicios de la agencia de los Estados Unidos para el Desarrollo
Internacional (USAID) este programa ha logrado avances importantes. Tiene como propósito dotar de los elementos de gestión ambiental municipal que faciliten los procesos y las actividades de forma tal que cumplan con sus misiones institucionales (Ministerio de Medio Ambiente y Recursos Naturales, República Dominicana, 2012). En temas puntuales en relación a la sostenibilidad y manejo de recursos naturales podemos mencionar los siguientes proyectos como consecuencia de avance en la gestión ambiental local:

- Programa de protección y conservación de las cuencas hidrográficas y especies amenazadas del municipio de Jardín/Colombia (Organización para la Cooperación y el Desarrollo Económico, OCDE, 2016). El uso excesivo e inadecuado de los recursos hídricos en Antioquia y en el suroeste de la región colombiana la cual presenta dificultades que deterioran a gran escala sus ecosistemas estratégicos. El proyecto tiene como objetivo instituir los acuerdos recíprocos por agua, que funcionan como incentivos para que los propietarios y administradores de los predios rurales desarrollen procesos productivos con alternativas de menor impacto ambiental.

- Gestión comunitaria del agua y desarrollo territorial en Waslala/Nicaragua (Organización para la Cooperación y el Desarrollo Económico, OCDE, 2016). La principal problemática en las zonas de es el recurso hídrico que es escaso. Aunque aproximadamente el $75 \%$ de los hogares tienen acceso al vital líquido, un $25 \%$ debe consumir agua de los arroyos o pozos, que no reúne condiciones de potabilidad. El proyecto pretende articular el proceso de ejecución y seguimiento del plan de desarrollo territorial en Waslala a través de la creación y fortalecimiento de un espacio de concertación que en este momento se ha definido como Grupo de Acción Local (GAL).

- Fortalecimiento de capacidades para la gestión ambiental de los residuos sólidos municipales en el distrito de Yauyos/Perú (Organización para la Cooperación y el Desarrollo Económico, OCDE, 2016). El proyecto nace a partir de dos insuficiencias observadas en el distrito de Yauyos: 1) las escasas capacidades técnicas de los trabajadores municipales y de la población en general para la gestión ambiental de los recursos sólidos, y 2) la falta de institucionalidad ambiental y de articulación entre la municipalidad, la población y las instituciones público y privadas para la planificación de estrategias que conlleven al buen manejo de los recursos sólidos. Al fortalecer las capacidades municipales en materia de limpieza pública y promover la institucionalidad 
ambiental, el proyecto pretende incrementar la calidad de vida de la comunidad y promover un ambiente libre de contaminación ocasionada por el mal manejo de los residuos sólidos.

\section{METODOLOGÍA}

\section{Tipo de estudio}

La investigación fue realizada con enfoque de tipo cualitativo orientada en comprender y profundizar los fenómenos explorándolos desde la perspectiva de los participantes en un ambiente natural y en relación con el contexto (Hernández Sampieri, 2003).

Se utilizó la recolección y el análisis de datos, al contestar preguntas por los sujetos de la de investigación. El alcance de la investigación fue de tipo descriptivo y a su vez se le agregaron características del tipo explicativo ya que en la investigación se midió y recolectaron datos sobre la situación actual de las unidades ambientales municipales del departamento de El Salvador.

Basada en el método científico, la investigación busca determinar la situación actual de las unidades ambientales municipales tanto en infraestructura, equipo y personal adecuado, como la existencia del marco normativo municipal para los aspectos ambientales determinantes, pero especialmente determinar la existencia y puesta en práctica de los instrumentos de gestión administrativos en materia ambiental en los municipios del departamento de Santa Ana.

\section{Población}

Personas entre funcionarios públicos municipales, usuarios y expertos en medio ambiente que tengan responsabilidad, interés, conocimiento e injerencia en temas de gestión ambiental local.

\section{Muestra}

De la población se seleccionaron 3 grupos importantes, de los cuales los funcionarios de las unidades brindaron la información oficial:

- Funcionarios, especialmente los jefes de las unidades ambientales municipales de los municipios de la ciudad de Santa Ana:
Los municipios son agrupados en los siguientes distritos:

- Distrito de Santa Ana (formado por los municipios: Santa Ana, Texistepeque, Coatepeque y El Congo). De los cuales todos aportaron los datos necesarios para el análisis, a excepción de Texistepeque.

- Distrito de Metapán (formado por los municipios: Metapán, Masahuat, San Antonio Pajonal, Santiago de la Frontera y Santa Rosa Guachipilín). Entre estos Metapán fue el municipio que más apertura mostró en facilitar la información necesaria.

- Distrito de Chalchuapa (formado por los municipios: Chalchuapa, Candelaria de la Frontera, El Porvenir y San Sebastián Salitrillo). De estos todos mostraron apertura para compartir la información necesaria.

Nota: La información presentada es de los municipios de cada distrito que presentaron mayor apertura a compartir y facilitar la información.

\section{Técnicas e instrumentos de investigación}

- Ficha propia de análisis de la situación actual de las unidades ambientales, con la finalidad de registrar hallazgos importantes que por medio de la observación se lograron determinar durante las visitas de campo.

- Entrevista con base en un cuestionario de preguntas abiertas y cerradas entregado a las personas seleccionadas de la muestra, los funcionarios de las unidades ambientales, con la finalidad de registrar su opinión y experiencias en cada uno de los aspectos relevantes seleccionados para evaluar las competencias de las unidades ambientales.

\section{Procedimiento y ejecución}

Aplicación de instrumentos:

- Realización de entrevistas: 10 preguntas abiertas y cerradas se aplicarán a los funcionarios de los municipios seleccionados que forman parte de nuestra muestra. La entrevista es el instrumento idóneo ya que el número de funcionarios es menor, se podrá tener más tiempo de intercambiar planteamientos con el objeto de determinar eficiencia de unidades ambientales, al igual que con el expertose realizó una entrevista breve de 10 preguntas abiertas. 
- La ficha es un instrumento que permitirá transmitir los datos que por observación serán recabados con el objetivo de establecer las condiciones de las unidades ambientales en temas de normativa ambiental local, infraestructura y personal idóneo, así como de los instrumentos que forman un sistema de gestión ambiental.

\section{RESULTADOS}

Culminando con el cumplimiento del objetivo general de la investigación: realizar una evaluación de la gestión ambiental local en los municipios del departamento de Santa Ana para determinar la capacidad de las mismas. Basados en los resultados obtenidos en las entrevistas realizadas, así como apoyado en los resultados de las fichas propias de análisis en las cuales se evaluaba cada uno de los criterios relevantes para la gestión ambiental municipal, se llega a la conclusión de que las unidades de medio ambiente no cuentan con las capacidades necesarias para enfrentar la misión y los desafíos que la ley delega en materia ambiental. Los resultados de los criterios evaluados son los siguientes:

\section{Criterio 1: Nivel de capacitación}

Muestra el nivel de capacitación de las jefaturas de las unidades ambientales analizadas, teniendo como opciones: aprendizaje empírico, bachiller, técnico, licenciatura, ingeniera y maestría. De los resultados, observamos que un $33 \%$ de la muestra poblacional no posee estudios universitarios, lo cual es preocupante debido a las responsabilidades que conlleva la jefatura ambiental; pero un 33 \% tienen estudios de posgrado en temas medioambientales, lo que les daría una visión más estratégica del área; este último porcentaje lo encontramos en los municipios de Metapán y Coatepeque.

Tabla y gráfica 1. Nivel de capacitación de la jefatura de las unidades ambientales municipales

\begin{tabular}{|l|l|}
\hline Empírico & 0 \\
\hline Bachiller & 2 \\
\hline Técnico & 0 \\
\hline Licenciatura & 1 \\
\hline Ingeniería & 1 \\
\hline Maestría & 2 \\
\hline
\end{tabular}

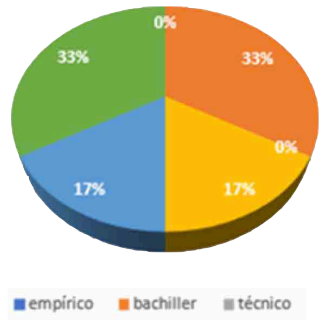

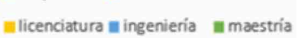

\section{Criterio 2: Infraestructura}

En la tabla y gráfica 2 se muestra la opinión de los jefes de las unidades ambientales en cuanto a la infraestructura institucional para realizar de forma adecuada sus actividades, el $83 \%$ opinó que la infraestructura es la adecuada. Cabe destacar que en las visitas de campo y según la ficha propia de análisis se logró observar que el municipio que posee la mejor infraestructura para el desarrollo de las actividades es el municipio de Metapán.

Tabla y gráfica 2. Infraestructura institucional de las unidades ambientales municipales

\begin{tabular}{|l|l|}
\hline Buena & 5 \\
\hline Mala & 1 \\
\hline
\end{tabular}

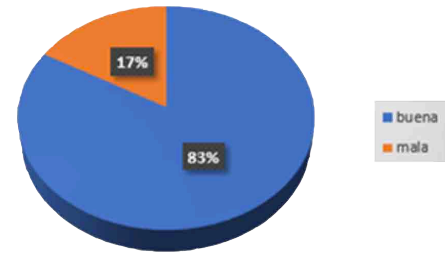

Fuente: elaboración propia.

\section{Criterio 3: Material y equipo}

En la tabla y gráfica 3 se muestra la opinión acerca del material y equipo necesario en las unidades ambientales. El $50 \%$ de los entrevistados opina que es el adecuado, se refieren al equipo administrativo, en cuanto al equipo especializado todos mencionaron tener deficiencias y falta del mismo. De los resultados de la ficha propia de observación obtenemos $100 \%$ los municipios participantes manifestaron deficiencias o falta del equipo técnico especializado lo cual es preocupante ya que no pueden realizar ningún tipo de prueba o medición de la calidad de los elementos ambientales.

Tabla y gráfica 3. Material y equipo de las unidades ambientales municipales

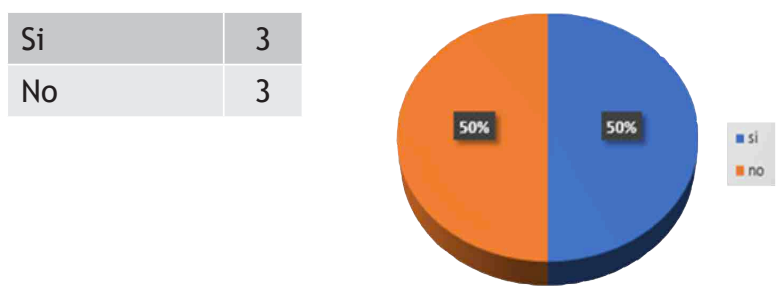

Fuente: elaboración propia. 


\section{Criterio 4: Ordenanzas medioambientales}

En la tabla y gráfica 4 podemos ver la opinión de las jefaturas de cada unidad referente a la existencia de las ordenanzas medioambientales en positivo en un $87 \%$, pero se refieren ordenanzas de carácter generales. En los resultados de la ficha de observación la mayoría manifestó de la inexistencia ordenanzas específicas en materia ambiental pues solo poseen una ordenanza general. Se puede concluir que este resultado es muy preocupante ya que las municipalidades no cuentan con este instrumento jurídico para regular actividad en el municipio.

Tabla y gráfica 4. Ordenanzas ambientales municipales

\begin{tabular}{|l|l|}
\hline $\mathrm{Si}$ & 5 \\
\hline No & 1 \\
\hline
\end{tabular}

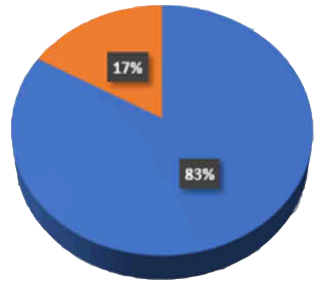

Fuente: elaboración propia.

\section{Criterio 5: Plan de gestión ambiental municipal}

En la tabla y gráfica 5 podemos observar el resultado de la consulta de la existencia de un plan de gestión ambiental municipal. El $83 \%$ manifestó poseer un plan de gestión ambiental, pero gracias a la ficha de análisis se puede determinar que al igual que en la pregunta referente a las ordenanzas ambientales hay una confusión entre un plan municipal que es de carácter general y operativo y el plan de gestión ambiental, que es de carácter específico y estratégico en materia ambiental. La confusión podría darse por el desconocimiento y falta de preparación técnica de las jefaturas ambientales municipales participantes, el resultado, por lo tanto, es engañoso ya que el $100 \%$ de las municipalidades no presentó el documento cuando fue requerido.

Tabla y gráfica 5. Plan de gestión ambiental municipal

\begin{tabular}{|l|l|}
\hline Si & 5 \\
\hline No & 1 \\
\hline
\end{tabular}

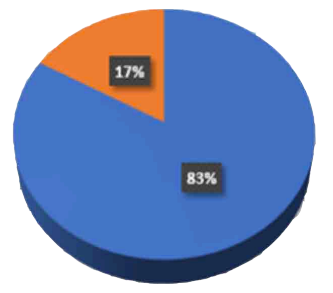

Fuente: elaboración propia.

\section{Criterio 6: Diagnósticos ambientales}

Las gráficas y tablas de la 6 a la 11 se refieren a los resultados de la existencia de los diagnósticos ambientales de cada municipio en los aspectos ambientales determinados: tratamiento de aguas residuales municipales, contaminación de los ríos y lagos del municipio, áreas naturales protegidas del municipio, uso de suelos del municipio, estado de los desechos sólidos municipales y estado del rastro municipal.

Los resultados son los siguientes:

a. Tratamiento de aguas residuales municipales: El $83 \%$ de los municipios visitados no cuenta con un diagnóstico ni datos específicos de las aguas residuales como fuentes de contaminación, ubicación geográfica de las fuentes de contaminación, tipo de contaminantes, etc. Solo el $17 \%$ de los municipios tiene un inicio de un diagnóstico cabe mencionar que en este caso es representado por el municipio de Metapán.

Tabla y gráfica 6. Diagnóstico de tratamiento de aguas residuales municipales

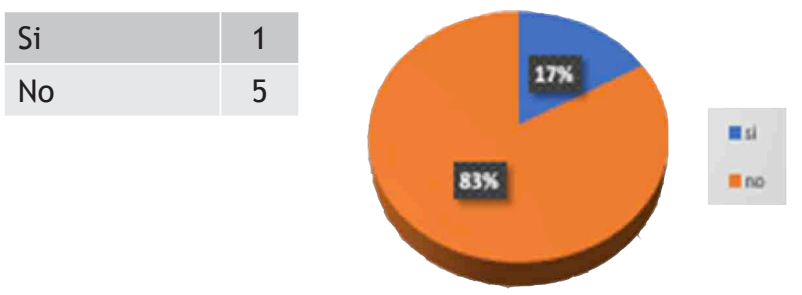

Fuente: elaboración propia.

b. Contaminación de los ríos y lagos del municipio: El recurso hídrico es un eje de desarrollo estratégico para cualquier comunidad. Es de vital importancia iniciar con su protección y adecuado manejo, el primer paso de esta gestión debería ser un diagnóstico de la situación actual de las fuentes de agua municipales. Como podemos observar en la tabla y gráfica 7 . El $67 \%$ de las unidades manifestó tener un diagnóstico de la contaminación de ríos y lagos de su municipio, cabe destacar que durante las visitas en general no pudieron determinar el nivel de contaminación ni las fuentes de la misma. 
Tabla y gráfica 7. Diagnóstico de contaminación de los ríos y lagos del municipio

\begin{tabular}{|l|l|}
\hline Si & 4 \\
\hline No & 2 \\
\hline
\end{tabular}
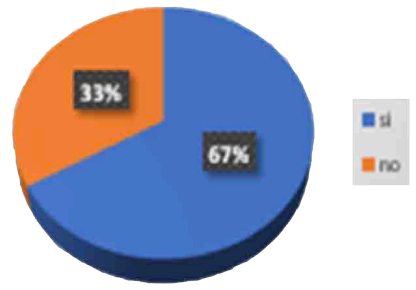

Fuente: elaboración propia.

c. Áreas naturales protegidas del municipio: Tener un diagnóstico de áreas naturales protegidas es importante porque son partes del territorio nacional legalmente establecidas con el objeto de posibilitar la conservación, el manejo sostenible y restauración de la flora y la fauna silvestre, recursos conexos y sus interacciones naturales y culturales, que tengan alta significación por su función o sus valores genéticos, históricos, escénicos, recreativos, arqueológicos y protectores, de tal manera que preserven el estado natural de las comunidades bióticas y los fenómenos geomorfológicos únicos. En la tabla y gráfica 8 se puede observar que el $67 \%$ de los municipios no poseen un diagnóstico de áreas protegidas, lo cual es un signo de una gestión ambiental ineficiente, al no contar con un diagnóstico no podrán hacer esfuerzos estructurados de conservación y protección ya que se desconocen las características propias de la región.

Tabla y gráfica 8. Diagnóstico de áreas naturales protegidas del municipio

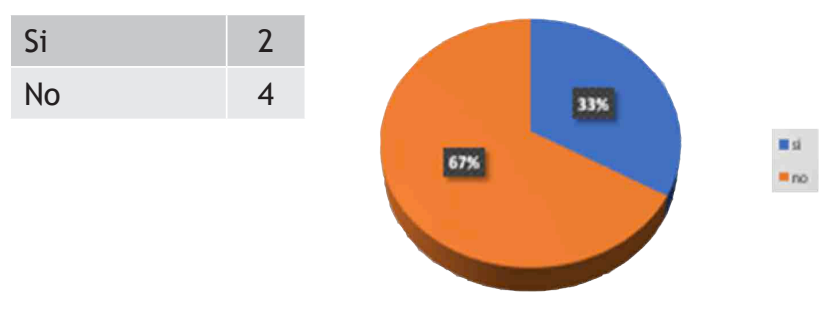

Fuente: elaboración propia.

d. Uso de suelos municipal: El uso del suelo comprende las acciones, actividades e intervenciones que realizan las personas sobre un determinado tipo de superficie para producir, modificarla o mantenerla. Este diagnóstico es de vital importancia ya que determina la vocación del territorio, abarca la gestión y modificación del medio ambiente natural para convertirlo en terreno agrícola: campos cultivables, pastizales, o asentamientos humanos. En la gráfica y tabla 9 se muestran los resultados a la pregunta de si existe un diagnóstico de uso de suelo municipal al que la mitad de los municipios visitados respondieron afirmativamente.

Tabla y gráfica 9. Diagnóstico de uso de suelos del municipio

\begin{tabular}{|l|l|}
\hline Si & 3 \\
\hline No & 3 \\
\hline
\end{tabular}

Fuente: elaboración propia.

e. Desechos sólidos: Un diagnóstico del manejo de desechos sólidos es importante porque es la gestión de los residuos, la recogida, el transporte, tratamiento, reciclado y eliminación de los materiales de desecho. El término generalmente se refiere a los materiales producidos por la actividad humana, y, en general, para reducir sus efectos sobre la salud y el medio ambiente.

La gráfica y tabla 10 muestran que el $67 \%$ de los municipios visitados no posee un registro de los desechos sólidos municipales y su impacto en el ambiente lo cual es muy preocupante.

Tabla y gráfica 10. Diagnóstico de desechos solidos del municipio

\begin{tabular}{|l|l|}
\hline Si & 2 \\
\hline No & 4 \\
\hline
\end{tabular}
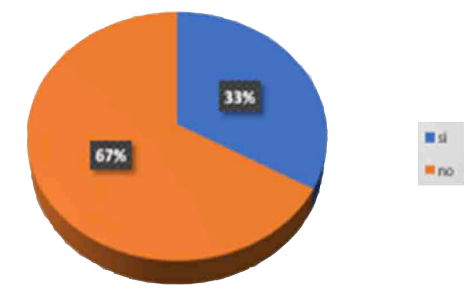

Fuente: elaboración propia.

f. Rastro municipal: Un rastro es un establecimiento destinado a la matanza de animales para el consumo humano, cuyos requisitos deben cumplirse en función de la legislación vigente, y tiene como objetivo principal proporcionar instalaciones adecuadas para que los particulares realicen el sacrificio de animales mediante los procedimientos adecuados. En la tabla y gráfica 11 observamos que el $100 \%$ de los municipios no posee ningún estudio del estado e impacto que el rastro municipal causa en el ambiente, tener un estudio sería lo más adecuado. 
Tabla y gráfica 11. Diagnóstico de rastro municipal

\begin{tabular}{|l|l|}
\hline Si & 0 \\
\hline No & 6 \\
\hline
\end{tabular}

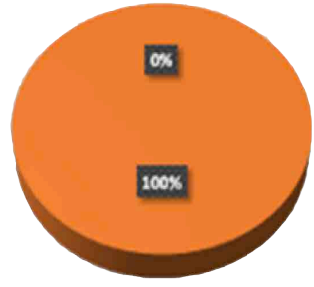

Fuente: elaboración propia.

\section{Criterio 7: Instrumentos de sistema de gestión ambiental}

En la visitita de campo se preguntó por la existencia de los instrumentos básicos que conforman un SGA, entre los que tenemos: política ambiental municipal, plan de gestión ambiental municipal, objetivos ambientales municipales, principios de acción bien definidos respecto al medioambiente, identificación para los aspectos e impactos ambientales relevantes, y charlas de sensibilización y educación ambiental. En las tablas y gráficas 12 y 13, como podemos observar, el $50 \%$ de las municipalidades manifestó poseer estos instrumentos, cabe destacar que durante la visita ninguna municipalidad manifestó poseerlo, sino que tenían algún documento como anteproyecto en el mejor de los casos, en general la confusión puede ser adrede o por falta de capacitación técnica de las jefaturas de las unidades.

Tabla y gráfica 12. Instrumento política ambiental municipal

\begin{tabular}{|l|l|}
\hline Si & 3 \\
\hline No & 3 \\
\hline
\end{tabular}

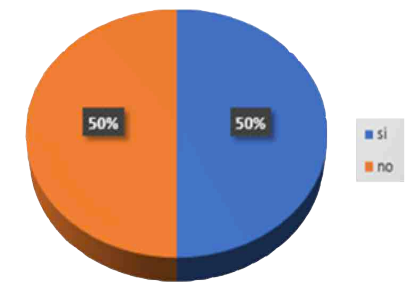

Fuente: elaboración propia.

Tabla y gráfica 13. Instrumento plan de gestión ambiental municipal

\begin{tabular}{|l|l|}
\hline Si & 3 \\
\hline No & 3 \\
\hline
\end{tabular}

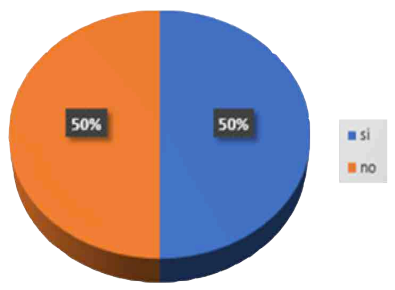

Fuente: elaboración propia.

\section{Criterio 8. Nivel de capacitación medio ambiental}

La educación ambiental desde la perspectiva local como una vía hacia la gestión sostenible de nuestros municipios, siendo un eje transversal en la concepción de las políticas que lleven a la satisfacción de las necesidades económicas, sociales y medioambientales, respetando al mismo tiempo la integridad cultural, y los procesos ecológicos esenciales, diversidad biológica y los sistemas que sostienen la vida. Según los resultados, podemos observar que el $67 \%$ de las unidades ambientales manifestaron la necesidad de capacitación en temas ambientales.

Tabla y gráfica 14. Capacitación en temas de medio ambiente

\begin{tabular}{|l|l|}
\hline Si & 2 \\
\hline No & 4 \\
\hline
\end{tabular}

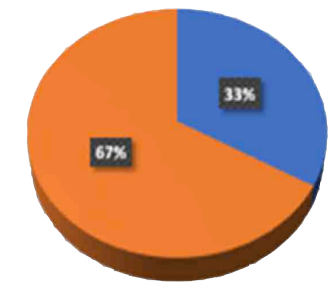

Fuente: elaboración propia.

Discusión, referida al propósito principal de la investigación

La investigación buscaba determinar de la situación actual de las unidades ambientales municipales en aspectos estratégicos necesarios para realizar la función que la ley manda, para lo cual se determinaron los siguientes criterios: capacitación del personal, infraestructura, equipo y personal adecuado, como la existencia del marco normativo municipal en materia ambiental, los aspectos ambientales determinantes. Especialmente, el trabajo buscaba determinar los instrumentos de gestión administrativos para formar un sistema de gestión ambiental.

Los municipios han realizado esfuerzos en los últimos años para poder realizar las actividades que la ley les otorga, pero la falta de capacitación del personal, la falta de equipo técnico, recursos, principalmente normativas, y el marco administrativo, hacen que estos esfuerzos sean poco efectivos. Resulta preocupante la ausencia de elementos tan básicos como los diagnósticos en las distintas áreas ambientales del municipio, como podemos ver en el criterio 6 y en las gráficas y tablas de la 6 a la 11. Preocupa, asimismo, el desconocimiento y la falta de los instrumentos administrativos para gestionar el municipio en el tema ambiental, como podemos ver en el criterio 7 y en 
las tablas y gráficas 12 y 13 . La mayoría confundía, por desconocimiento y falta de capacitación, los programas y los planes operativos con los instrumentos de gestión ambientales. Es preocupante la falta de instrumentos de gestión local para enfrentar los desafíos en materia ambiental a que se ve expuesto el departamento. En la tabla 15 se muestran algunos problemas sectoriales recientes.

Tabla 15. Noticias de problemática sectorial relacionada con temas ambientales del departamento de Santa Ana

\section{Denuncian tala en cerro Santa Lucía}

La tala en el cerro, eleva el riesgo de que se produzcan derrumbes o deslaves, debido a que desde hace varios años el lugar fue catalogado por las autoridades correspondientes de la época como zona de riesgo, por la deforestación, poniendo en riesgo las viviendas de la zona (La Prensa Gráfica. El Salvador, 2014).

134 kilómetros de ríos contaminados por derrame de melaza

Unas 33 especies acuáticas murieron por la falta de oxígeno (El Diario de Hoy. El Salvador, 2016).

Salazar Romero (empresa constructora) debe compensar daños ambientales en Atiquizaya (UNES, La Unidad Ecológica Salvadoreña, 2015).

Ordenan no conectar aguas negras a manantial

El Juzgado Ambiental resolvió que la empresa constructora residencial Salazar Romero suspenda de forma total el desagüe de aguas negras sobre el acuífero (El Diario de Hoy. El Salvador, 2015).

FGR tras las pistas de contaminación en río

Las autoridades ingresaron a una tenería y comprobaron que los desechos del lugar son lanzados al río Chiguite sin recibir tratamiento (El Diario de Hoy. El Salvador, 2012).

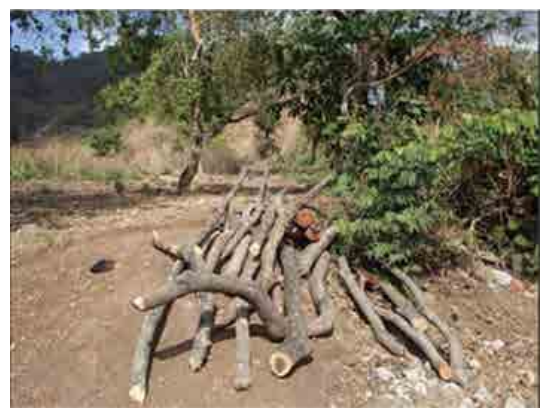

Foto: Deforestación en el cerro Santa Lucia. Foto de La Prensa Gráfica. El Salvador.

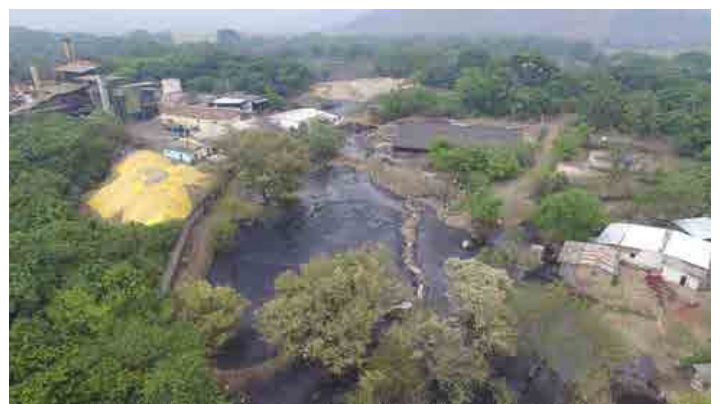

Derrame de melaza en el Ingenio La Magdalena. Foto de Mauricio Castro.

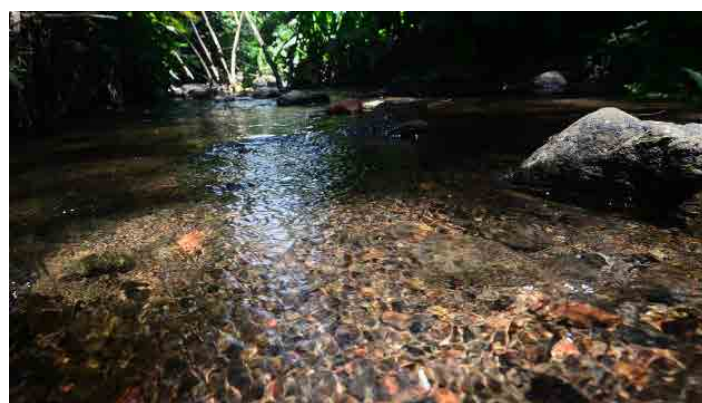

Manantial en Atiquizaya hacia el cual se realizará la conexión de aguas negras. Foto de Miguel Villalta.

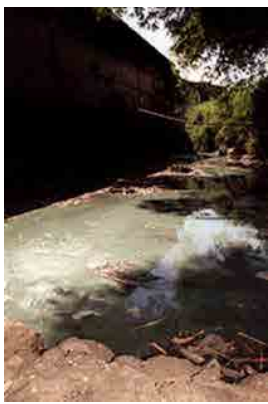

Desechos lanzados al río Chiguite sin recibir tratamiento. Foto de Wenceslao Martínez.

Fuente: elaboración propia. 
Podemos analizar los resultados y concluir en tres grandes áreas necesarias para el eficiente funcionamiento de las unidades ambientales locales:

\section{Objetivos y funciones (MARN, 2014)}

Las unidades ambientes municipales encargadas primordialmente de la gestión de las actividades que afectan al municipio en materia ambiental tienen objetivos como a) identificar competencias y actividades de las instituciones que requieran de acciones ambientales, y b) velar y promover el cumplimiento de la normativa jurídica sectorial de carácter ambiental.

Se puede concluir que esta área no se cumple de manera eficiente. Como vemos en los resultados en la tabla y gráfica 13 , el $50 \%$ de las unidades ambientales municipales visitadas no poseen un plan de gestión ambiental municipal. Si no se cuenta con un plan debidamente estructurado no se contará con objetivos estratégicos y los esfuerzos serán aislados y con pocos resultados.

\section{Personal, equipo e infraestructura (MARN, 2014)}

Para un buen desempeño de la Unidad Ambiental se considera necesario de 3 a 4 personas con los perfiles de un coordinador, uno o dos técnicos o un asistente. En cuanto al equipo, la UA debe contar con equipo de computación moderno.

Se puede concluir, como vemos en el criterio 1 , y en la tabla y gráfica 1 , que en relativo al personal en general no se cuenta con el personal idóneo, es lamentable que no exista un perfil de las aptitudes y capacidades que debe tener el futuro funcionario. Por otro lado, según los resultados de la investigación, en el criterio 2 , y en la tabla y gráfica 2 , el $83 \%$ está conforme con la infraestructura y el 50\% está conforme con el equipo. Cabe destacar que ninguna de las unidades tiene equipo especializado para medir aspectos en materia ambiental.

3. Acción de las unidades ambientales institucionales (MARN, 2014) a) Planificación y organización, b) coordinación y ejecución, c) control y seguimiento, d) evaluación del trabajo de la Unidad y metas alcanzadas.

En conclusión, en planificación y organización, sin un plan de gestión ambiental los esfuerzos serán insuficientes dispersos y poco efectivos. Según los resultados de la gráfica y tabla 13 , el $50 \%$ de las unidades ambientales municipales visitadas no poseen un plan ambiental, además, sin la existencia de un diagnóstico ambiental municipal no se pueden desarrollar actividades estratégicas en este sentido. Como se puede observar en la tabla y gráfica 15, más del $60 \%$ de las municipalidades no tiene diagnóstico ambiental del municipio.

Es necesaria la coordinación interinstitucional, compartir información con oNG ambientales y con las universidades nacionales para coordinar esfuerzos en el tema del desarrollo de instrumentos de gestión ambiental. Finalmente, se anexan mapas necesarios para comenzar a categorizar la información de manera estructurada y servir para realizar los primeros diagnósticos ambientales tan necesarios en nuestras municipalidades. La información para realizar estos mapas se encuentra en instituciones nacionales y oNG ambientales, pero por diversas razones no son utilizadas por las municipalidades.

La solucion a los conflictos sectoriales relacionados con el desequilibrio ambiental está en el diseño e implementación de un sistema de gestión ambiental (SGA) basado en las normativas nacionales e internacionales como la iso 14001. Una gestión ambiental exitosa debe responder a las características ambientales, sociales y económicas del municipio, el SGA tiene como objetivo principal la realización de un proceso de análisis, conceptualización y propuesta técnica para desarrollar un modelo que guíe la gestión ambiental municipal. Esta sería una planificación con objetivos específicos planes y programas estructurados a largo plazo en materia ambiental; como vimos, hay experiencias de éxito en Latinoamérica, países que han realizado esfuerzos serios en la gestión ambiental local (cuadros 1, 2 y 3). 
Cuadro 1. Mapa de áreas naturales protegidas

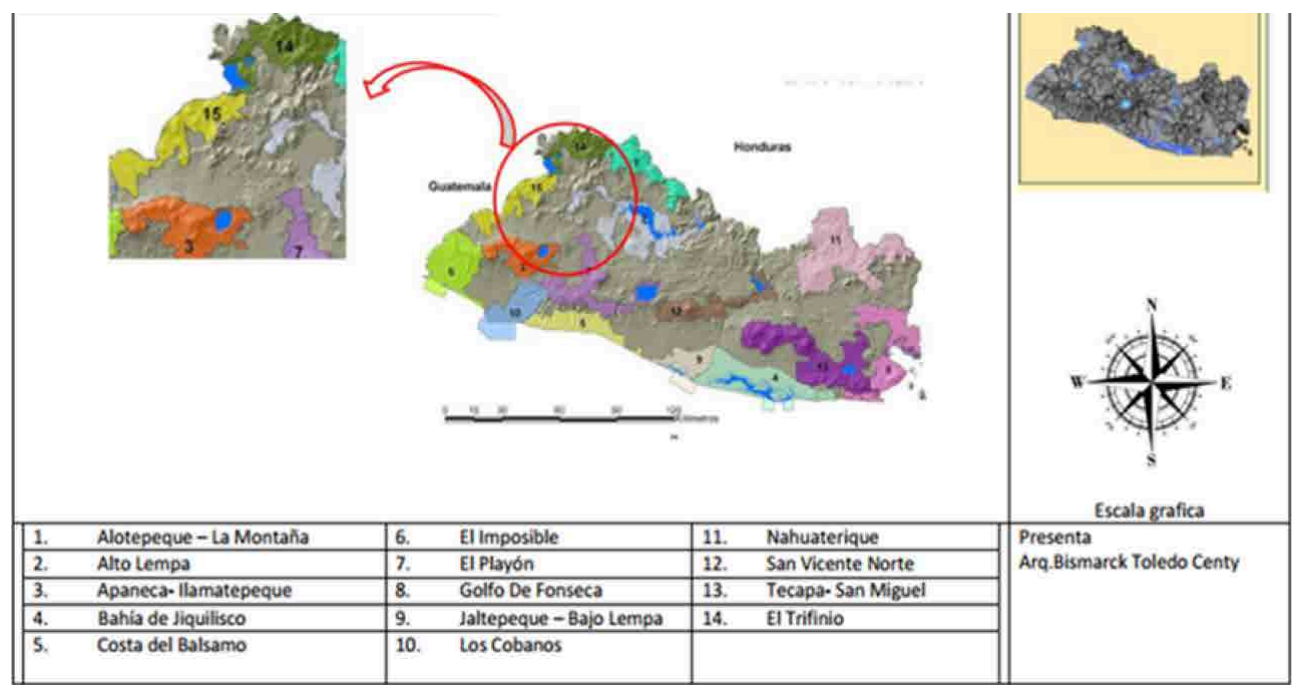

Fuente: elaboración propia.

Cuadro 2. Mapa de cobertura y uso de suelo. Municipio de Metapán

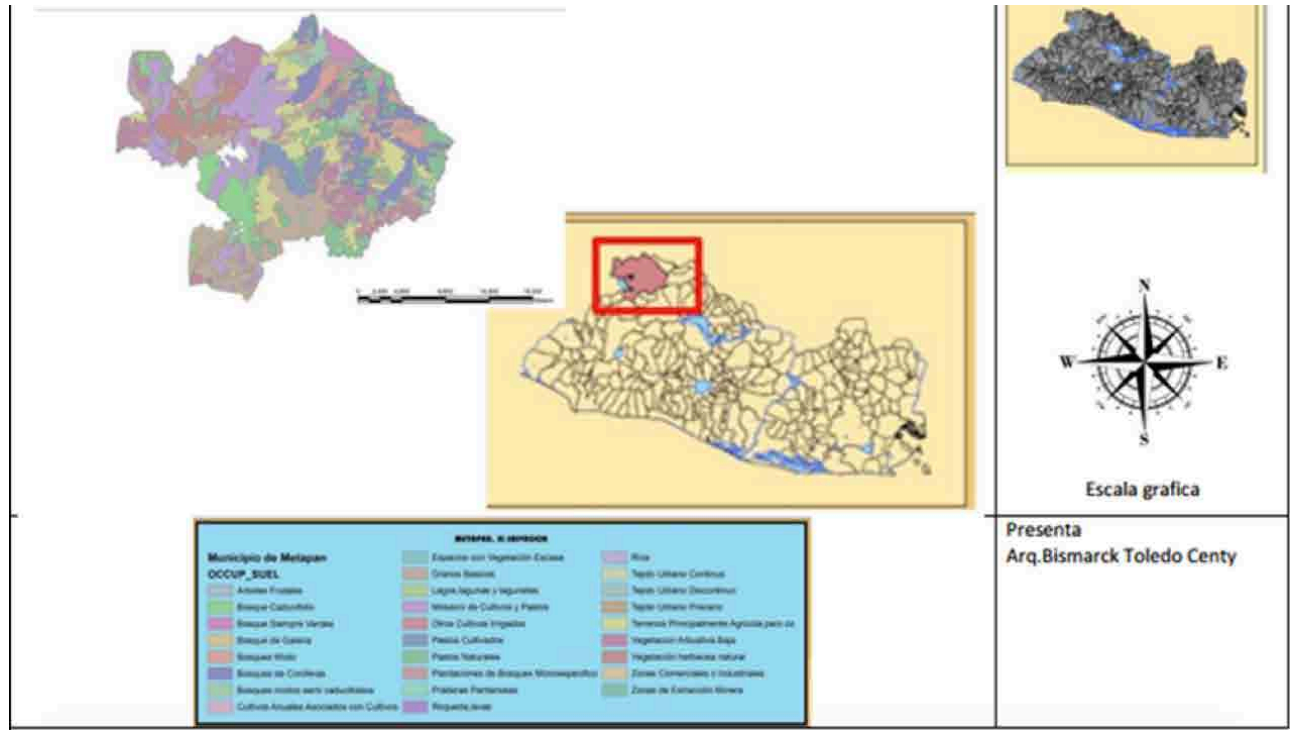

Fuente: elaboración propia. 
Cuadro 3. Mapa de hidrografía, áreas protegidas y relieve. Municipio de Santa Ana

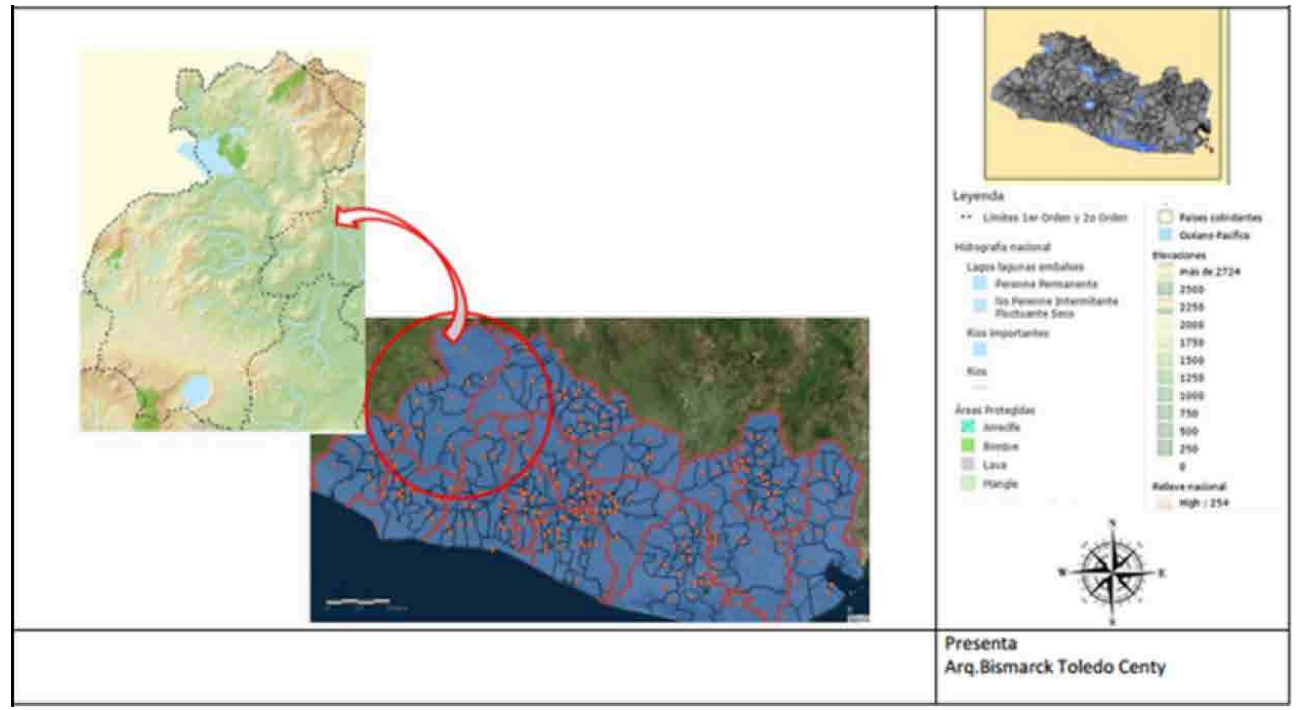

Fuente: elaboración propia.

\section{REFERENCIAS}

Asociación Chilena de Municipalidades, Fundación Friedrich Ebert. Santiago de Chile. (1995). Manual n. ${ }^{\circ}$ 5: Gestión Ambiental Municipal.

Comisión Mundial sobre Medio Ambiente y Desarrollo, onu. (1987). Nuestro Futuro Común, informe Brundtland.

El Diario de Hoy. El Salvador. (2012). FGR tras las pistas de contaminación en río. Obtenido de http: / /

conectar aguas negras a manantial. Obtenido de http://www.elsalvador.com/noticias/nacional/161639/ordenan-no-conectar-aguas-negras-a-manantial/

El Diario de Hoy. El Salvador. (2016). 134 kilómetros de ríos contaminados por derrame de melaza. Obtenido de http://www.elsalvador.com/noticias/nacional/188582/134-kilometros-de-rios-contaminados-por-derrame-de-melaza/

Hernández Sampieri, R. F. (2003). Metodología de la investigación. Mexico: McGraw-Hill.

International Organization for Standardization, Iso. (2015). ISO14001-Sistemas de gestión am- biental. Directrices generales sobre principios, sistemas y técnicas de apoyo.

Ministerio de Medio Ambiente y Recursos Naturales, MARN. (1998). Ley de Medio Ambiente. República de El Salvador.

Ministerio de Medio Ambiente y Recursos Naturales, MARN. (2014). Lineamientos para la conformación de las Unidades Ambientales Institucionales.

Ministerio de Medio Ambiente y Recursos Naturales, MARN. (2015). Guia metodologica para elaborar las herramientas de la Gestión ambiental municpal. guia MARN, Ministerio de Medio Ambiente y Recursos Naturales.
Ministerio de Medio Ambiente y Recursos Naturales, República Dominicana. (2012). Guía para la Gestión Ambiental Municipal.

Organización para la Cooperación y el Desarrollo Económico, OCDE. (2016). Compendio de buenas prácticas para el desarrollo local en américa latina.

Unidad Ecológica Salvadoreña, unEs. (2015). Salazar Romero debe compensar daños ambientales en Atiquizaya. Obtenido de http://www. unes.org.sv/2015/10/13/salazar-romero-debe-compensar-danos-ambientales-en-atiquizaya/ 


\title{
POTENCIAL LOGÍSTICO DE LOS PAÍSES DE AMÉRICA LATINA Y EL CARIBE: ANÁLISIS A PARTIR DE INDICADORES ECONÓMICOS, SOCIALES Y AMBIENTALES ${ }^{1}$
}

\author{
LOGISTIC POTENTIAL OF LATIN AMERICA AND THE CARIBBEAN COUNTRIES: AN ANALYSIS \\ BASED ON ECONOMIC, SOCIAL AND ENVIRONMENTAL INDICATORS \\ POTENCIAL LOGÍSTICO DOS PAÍSES DE AMÉRICA LATINA E O CARIBE: ANÁLISE A PARTIR \\ DE INDICADORES ECONÓMICOS, SOCIAIS E AMBIENTAIS \\ Elsa Cristina González La Rotta, Oswaldo González Yazo, Mauricio Becerra Fernández²
}

Fecha de recibido: Agosto 05 de 2017 | Fecha de aprobado: Octubre 02 de 2017

\section{Resumen}

El presente artículo analiza el potencial logístico de los países de América Latina y el Caribe, por medio de un indicador de calificación por país, generado a partir de un análisis cualitativo y cuantitativo de la correlación existente entre variables económicas, sociales y ambientales sobre los ingresos nacionales de las empresas del sector transporte y logística de cada país. Este indicador permite caracterizar los países de la región que presentan un escenario propicio para actividades relacionadas con logística. Se hace énfasis especial en la importancia de la sostenibilidad, particularmente desde la minimización del impacto ambiental, dada la naturaleza altamente contaminante de este tipo de operaciones.

Palabras clave: Potencial logístico, indicador, transporte, sostenibilidad, impacto ambiental.

1 Artículo de revisión.

2 Integrantes del Semillero de investigación Silos, ucc, Grupo GIP, Universidad Católica de Colombia. Correo electrónico: ecgonzalez@ucatolica.edu.co 